
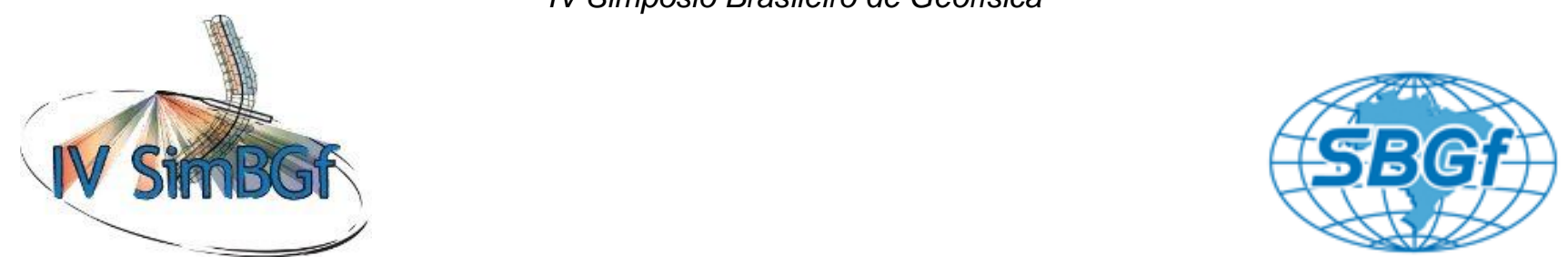

\title{
Análise Estatística de Perfis de Poço da Bacia do Jequitinhonha: Aplicações da Geometria Fractal
}

\author{
Misael Possidônio de Souza*, IGEO/UFBA \\ Amin Bassrei, CPGG/IGEO/UFBA e INCT - Geofísica de Petróleo
}

Copyright 2008, SBGf - Sociedade Brasileira de Geofísica

Este texto foi preparado para a apresentação no IV Simpósio Brasileiro de Geofísica, Belém, 14 a 17 de novembro de 2010. Seu conteúdo foi revisado pelo Comitê Técnico do IV SimBGf, mas não necessariamente representa a opinião da SBGf ou de seus do IV SimBGf, mas não necessariamente representa a opinião da SBGf ou de seus comerciais sem prévia autorização da SBGf.

\begin{abstract}
Fractal geometry is the field of Mathematics that studies the properties and behavior of fractals. A fractal is a geometric object that can be divided into several parts, each one similar to the original object. Fractal geometry has been used frequently to characterize and describe natural models. Its applications range from microscopic dimensions to the understanding of macroscopic processes. Following this principle, we proposed to study a set of well log data, collected in the Jequitinhonha basin (Brazil), through the methods of spectral analysis and R/S analysis in order to compute the Hurst exponent $H$. The knowledge of $H$ provides the computation of the fractal dimension, as well as to understand the complexity of layer sedimentation phenomenon. The application of fractal geometry concepts to the statistical characterization of well-logs indicate that their geophysical properties behave as fractals.
\end{abstract}

\section{Introdução}

A Geometria Fractal, introduzida por Benoit Mandelbrot, é uma geometria que trata de formas complexas, que tem sido aplicada nas diversas áreas do conhecimento, em especial na modelagem de fenômenos naturais. Um fractal é um objeto que pode ser dividido em partes semelhantes ao todo, propriedade que chamamos de auto-similaridade. A Figura 1 mostra a folha de samambaia, é um exemplo clássico de fractal encontrado na natureza.

Entretanto, o uso de fractais auto-similares como modelos não é aplicável para estudar séries que variam no tempo e espaço. O uso das funções fractais faz-se necessário para estudar estes fenômenos, das quais um tipo especial de função foi proposto por Mandelbrot \& Van Ness (1968), o chamado movimento fracionário browniano (mfb). O mbf apresenta um comportamento de escala estatístico ao invés da perfeita auto-similaridade, possuindo o espectro de potência $P$ inversamente proporcional à freqüência $f$ :

$$
P \propto \frac{1}{f^{b}},
$$

onde $\boldsymbol{b}$ é o coeficiente espectral. Quando $\boldsymbol{b}$ for igual a 0 , temos o chamado ruído branco, o qual possui pouca ou nenhuma correlação. Quando o coeficiente espectral $\boldsymbol{b}$ assume valores entre 0,5 e 1,0 , temos o ruído $1 / f$.

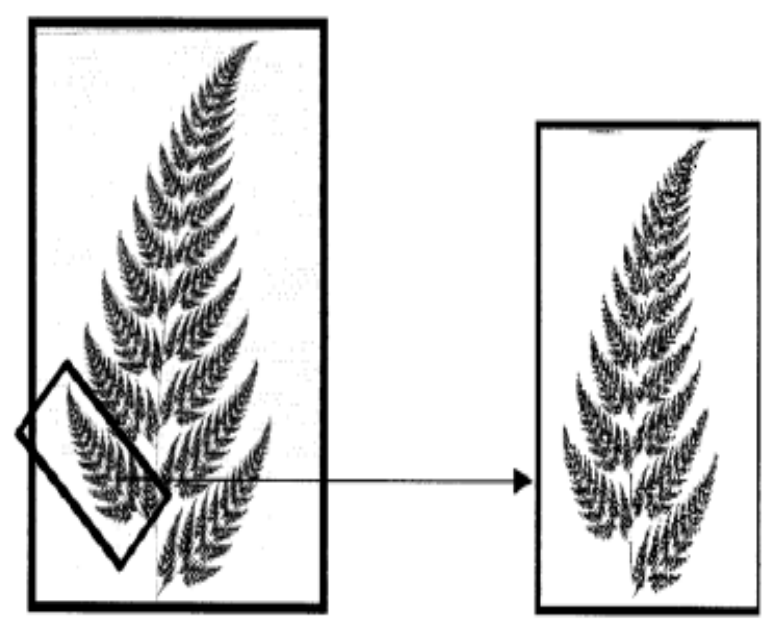

Figura 1 - A folha de samambaia é um exemplo clássico de fractal auto-similar (Retirado de Feder, 1989).

Uma técnica para estudar a correlação de longo alcance ou persistência de uma série é a Análise $\mathrm{R} / \mathrm{S}$, do inglês re-scaled analysis. $\mathrm{Na}$ análise $\mathrm{R} / \mathrm{S}$ se calcula 0 coeficiente de Hurst $\mathrm{Hu}$ (Hurst, 1957), parâmetro que define a persistência da série analisada. A persistência é a medida de quanto uma tendência local continua ao longo de toda a série.

Neste trabalho, mostramos alguns dos resultados obtidos da caracterização estatística de dados de poços da bacia do Jequitinhonha. As propriedades analisadas foram:

- emissão natural de raios ga0ma (GR),

- potencial espontâneo (SP),

- resistividade (ILD),

- condutividade (CILD),

- tempo de trânsito de onda P (DT), 
- densidade (RHOB) e

- $\quad$ porosidade neutrônica (PHIN).

Para cada perfil, calculamos os coeficientes $b$ e $H u$ os quais serão utilizados para obter informações estatísticas dos perfis analisados.

\section{Metodologia}

Para calcular o coeficiente espectral $b$ e o coeficiente de Hurst $H u$ utilizamos as técnicas da Análise Espectral e Análise R/S. A Análise Espectral descreve como a energia é distribuída nas faixas de freqüência ou número de onda. Fundamenta-se no princípio básico de Fourier, que diz que qualquer forma de onda pode ser representada como um somatório infinito de funções seno e cosseno, representado pelas séries de Fourier (Bracewell, 1986; Hsu, 1970).

Para as funções não periódicas e discretas, o tratamento matemático de Fourier é feito através da transformada discreta de Fourier, cujo cálculo pode ser efetuado de maneira mais rápida através de algoritmos conhecidos como transformada rápida de Fourier, ou FFT, do inglês Fast Fourier Transform. O espectro de potência $P$ da série é então calculado através das partes real e imaginária da transformada discreta de Fourier, via FFT. Aplicando $\log$ em ambos os lados da equação (1) teremos:

$$
\log P=\log k-b \log f
$$

onde o coeficiente espectral $b$ é igual ao coeficiente angular da reta ajustada através da regressão linear dos valores de $\log P$ e de $\log f$.

A Análise R/S ou Análise de Hurst foi desenvolvida por Hurst (1957) quando o mesmo estudava o comportamento do rio Nilo envolvido com a construção da barragem de Assuã. O problema era determinar a vazão ideal de armazenamento de água num reservatório para um fluxo de entrada aleatório. Sendo $y(z)$ a série espacial, a seqüência cumulativa $X(f(z), N)$ da série é calculada por:

$$
X(y(z), N)=\sum_{n=1}^{N}(y(z)-\mu)
$$

onde $\mu$ é a média aritmética da série. A variável $R(y(z), N)$ é a diferença entre o valor máximo e o valor mínimo dentro da seqüência cumulativa:

$$
R(y(z), N)=\max (X(y(z), N)-\min (X(y(z), N)
$$

Hurst concluiu que para diversos fenômenos era necessário normalizar a variável $R$ dividindo seu valor pelo seu desvio padrão $S$, as quais se relacionavam experimentalmente através de:

$$
R / S=k N^{H u},
$$

onde $H u$ é o coeficiente de Hurst. Aplicando $\log$ em ambos os lados da equação acima, teremos:

$$
\log R / S=\log k+H u \log N
$$

onde o coeficiente $\mathrm{Hu}$ é igual ao coeficiente angular da reta ajustada através da regressão linear dos valores de $\log R / S$ e de $\log N$.

O ruído branco possui coeficiente de Hurst igual a 0,5. A persistência da série ocorre para valores de $\mathrm{Hu}$ entre 0,5 e 1,0, e a anti-persistência ocorre para valores de $H u$ entre 0,0 e 0,5 . Os casos de persistência e antipersistência revelam uma componente de memória longa com componente aleatória na série, onde eventos passados se relacionam positivamente (persistente) ou negativamente (anti-persistente) com eventos futuros.

Essa abordagem foi sugerida por Leonard \& Kümpel (1998), que utilizaram em dados de poços do Projeto $\mathrm{KTB}$, Alemanha. Mais recentemente, Braga (2008), utilizou esta abordagem em dados climatológicos de Salvador, Bahia. Seus resultados são consistentes com outros autores que utilizaram análise R/S em dados climatológicos, tais como Miranda \& Andrade (1999) e Chierice (2003).

\section{Resultados}

O espectro de potência de cada perfil foi calculado por meio de periodogramas $P^{*}$ dado pela equação (Leonardi \& Kümpel, 1998):

$$
P^{*}=\frac{1}{2 \pi T_{M A X}}\left|\sum_{n=1}^{Z}(y(z)-\mu) e^{-i f z}\right|^{2},
$$

onde $T_{M A X}$ é o tamanho máximo da série, $\mu$ representa a média aritmética da série, $f$ é a freqüência ou número de onda, $i$ é a unidade imaginária, e $y(z)$ é a propriedade em função da profundidade. Para obter melhores resultados e reduzir a dispersão nos dados, Leonardi \& Kümpel (1998) recomendam a aplicação de uma suavização no espectro de potência antes mesmo da regressão línea. A suavização pode ser feita através da janela de Tukey-Hanning:

$$
P(f)=\frac{1}{4} P^{*}\left(f-f_{e}\right)+\frac{1}{2} P^{*}(f)+\frac{1}{4} P^{*}\left(f+f_{e}\right) .
$$

Primeiramente, aplicamos a Análise Espectral nos perfis sem nenhum processamento. As séries foram amostradas a cada 0,15 $\mathrm{m}$ totalizando em mais de 13500 amostras, com um intervalo de amostragem em freqüência calculado de $0,00047 \mathrm{~m}^{-1}$. Selecionamos o intervalo de $1380 \mathrm{~m}$ a $3500 \mathrm{~m}$ em profundidade para poder comparar os resultados entre cada perfil e entre técnicas diferentes. A Tabela 1 ilustra as propriedades físicas registradas, suas respectivas unidades físicas e as siglas aqui utilizadas para representar cada perfil. A 
Figura 2 mostra os perfis de poço utilizados neste trabalho.

Para todos os perfis, o espectro de potência se relacionou com a freqüência segundo uma lei de potência. As Figuras 3 e 4 mostram resultados da Análise Espectral para alguns perfis, no caso o tempo de trânsito da onda $P$ (DT) e o potencial espontâneo (SP). Em ambas os casos utilizamos o método dos mínimos quadrados (MMQ) para a regressão linear dos valores de $\log P$ e $\log f$.

Para aplicar a Análise R/S, utilizamos o algoritmo adaptado de Braga (2008), que utilizou esta abordagem em dados climatológicos de Salvador, Bahia. A parte prática desta técnica consiste na escolha dos valores dos tamanhos $N$ das sub-séries e dos pontos de partida $n$. Os pontos de partidas são necessários para evitar a sobreposição de amostras garantindo assim a aleatoriedade da variável $R / S$. O algoritmo recebe os valores da série de entrada, dos tamanhos de cada subsérie $N$ e dos pontos de partida $n$, para depois calcular a variável $R / S$ em função de cada tamanho $N$ escolhido. As Figuras 5 e 6 mostram os resultados para as mesmas variáveis para as quais foram mostrados os resultados. Mais uma vez, utilizamos o MMQ para a regressão linear entre os valores de $\log R / S$ e $\log N$. Nota-se 0 comportamento linear, o qual também foi observado nos demais perfis, não mostrados aqui por limitação de espaço. Os valores dos coeficientes $b$ e $H u$ encontram-se na Tabela 2 cinco perfis: emissão natural de raios gama, potencial espontâneo, resistividade, condutividade e tempo de transito da onda $\mathrm{P}$.

\section{Discussão e Conclusões}

O principal objetivo deste trabalho é caracterizar e descrever a estatística de séries espaciais em dados de poços, os quais podem ser bem modelados por classes de funções fractais, como as funções movimento fracionário browniano (mfb) e ruído $1 / f$, que exibem um comportamento de escala estatístico ao invés de perfeita auto-similaridade, como o floco de neve e a folha de samambaia.

O espectro de potência $P$ das propriedades decaiu com a potência do número de onda $f$, com valores do coeficiente espectral $b$ variando entre 1,5 e 2,5 para os perfis com espectro de potência suavizado, o que nos permite dizer que as diferentes propriedades subjacentes se comportam estatisticamente como o movimento fracionário browniano. Essa classe de funções é basicamente não estacionária e não gaussiana, além de possuir auto-afinidade e uma boa correlação. Estes resultados foram diferentes de trabalhos realizados com dados de poços de outras regiões. Leonard \& Kumpel (1998) encontraram valores de $b$ entre 0,5 e 1,5 para o conjunto de perfis de poços da KTB, tendo, portanto, um comportamento de ruído $1 / f$ o qual possui correlação intermediária entre o mfb e o ruído branco.
Estes autores afirmam ter aplicado uma filtragem nos dados para remover tendências lineares causadas pelo aumento da temperatura e pressão com a profundidade, o que não foi realizado nesta etapa deste trabalho.

Os valores do coeficiente de Hurst $H u$ variaram entre 0,67 e 0,73 , indicando persistência nas séries analisadas, fato já observado por outros autores. O perfil SP foi o único que obteve o coeficiente de Hurst maior que 1,0, o que não está de acordo com a analise R/S, fato ainda não observado na literatura e outros trabalhos. Assim um estudo diferenciado para o perfil SP torna-se necessário. Alguns efeitos que costumam ocorrer na análise R/S como efeitos de transiente inicial e efeito de ciclicidade não foram observados. Valores de $H u$ diferentes de 1,0 também são indicativos de auto-afinidade presente nos dados, o que confirma os resultados encontrados pela análise espectral, mostrando que diferentes métodos de análise dão os mesmos resultados, um importante fato para os sistemas complexos.

Nenhuma descorrelação $(H u=0,5)$ foi observada, levando-nos a afirmar que as propriedades analisadas possuem uma estatística bem correlacionada, o que geologicamente pode ser entendido como os processos de deposição sedimentar da bacia ocorreram de maneira a conservar características verticais estatísticas nas propriedades físicas, ao longo de várias escalas de observação, nas proximidades do intervalo analisado.

\begin{tabular}{|c|l|c|}
\hline Perfil & \multicolumn{1}{|c|}{ Propriedades física } & Unidade \\
\hline GR & Emissão natural de raios gama & UAPI \\
\hline SP & Potencial Espontâneo & $m V$ \\
\hline ILD & Resistividade & Ohm.m \\
\hline CILD & Condutividade & $m S . m$ \\
\hline DT & Tempo de transito da onda P & $m s$ \\
\hline
\end{tabular}

Tabela 1 - Perfis geofísicos e as propriedades correspondentes analisadas neste trabalho.

\begin{tabular}{|c|c|c|}
\hline Perfil & $\begin{array}{c}\text { Coeficiente } \\
\text { espectral } b\end{array}$ & $\begin{array}{c}\text { Coeficiente de } \\
\text { Hurst } \mathrm{Hu}\end{array}$ \\
\hline GR & 2,05 & 0,67 \\
\hline SP & 1,57 & 1,08 \\
\hline ILD & 2,28 & 0,66 \\
\hline CILD & 2,49 & 0,74 \\
\hline DT & 2,73 & 0,66 \\
\hline
\end{tabular}

Tabela 2 - Valor do coeficiente espectral e do coeficiente de Hurst dos perfis analisados neste trabalho. 


\section{Agradecimentos}

Os autores agradecem à FINEP pelo apoio à Rede CTPETRO de Geofísica de Exploração - Rede 01 - Fase 3 - "Imageamento do Talude Continental" e também agradecem ao LAGEP/CPGG/UFBA pela utilização dos recursos computacionais. Misael Possidônio de Souza agradece à bolsa de Iniciação Científica do CNPq relacionado ao mesmo projeto, e também agradece ao ANP/PRH-08 pela atual bolsa de graduação. Amin Bassrei agradece ao CNPq pela bolsa de produtividade de pesquisa.

\section{Referências}

Beer, R., 1994. Extensão da Análise R/S para Caracterização Geoestatística Local e Regional de Reservatórios de Hidrocarbonetos. Dissertação de Mestrado. Universidade Estadual de Campinas, Brasil.

Bracewell, R. N., 1986. The Fourier Transform and its Applications. McGraw-Hill, New York, USA.

Braga, B. M., 2008. Análise da Persistência de Séries Temporais em Dados Climatológicos de Salvador-Bahia. Trabalho de Graduação. Universidade Federal da Bahia, Brasil.

Chierice, R. A. F., 2003. O Uso de Wavelets na Determinação do Expoente de Hurst de uma Série
Temporal Diária de Chuvas do Município de Araras - SP de 1955-2000. Dissertação de Mestrado. Universidade Estadual Paulista Júlio de Mesquita Filho, Rio Claro, Brasil.

Feder, J., 1989, Fractals. Plenum Press, New York, USA.

Hewett, T. A., 1986, Fractal distributions to reservoir heterogeneity and their influence on fluid transport. SPE Annual Technical Conference and Exhibition, New Orleans, p. 67-82, SPE 15386.

Hsu, H. P., 1970, Fourier Analysis. Simon and Schuster, New York, USA.

Hurst, H. E., 1957. A suggested statistical model of some time series which occur in nature, Nature, vol. 180, p. 494-494.

Leonardi, S. \& Kümpel, H., 1998. Variability of the geophysical log data and signature of crustal heterogeneities at the KTB. Geophysical Journal International, vol. 135, p. 964-974.

Mandelbrot, B. \& Van Ness, J., 1968. Fractional Brownian motions, fractional noises and applications. SIAM Review, vol. 10 , p. $422-437$.

Miranda, J. G. V., Andrade, R. F. S., 1999. Rescaled range analysis of pluviometric records in northeast Brazil. Theoretical and Applied Climatology, vol. 63, p. 79-88. 


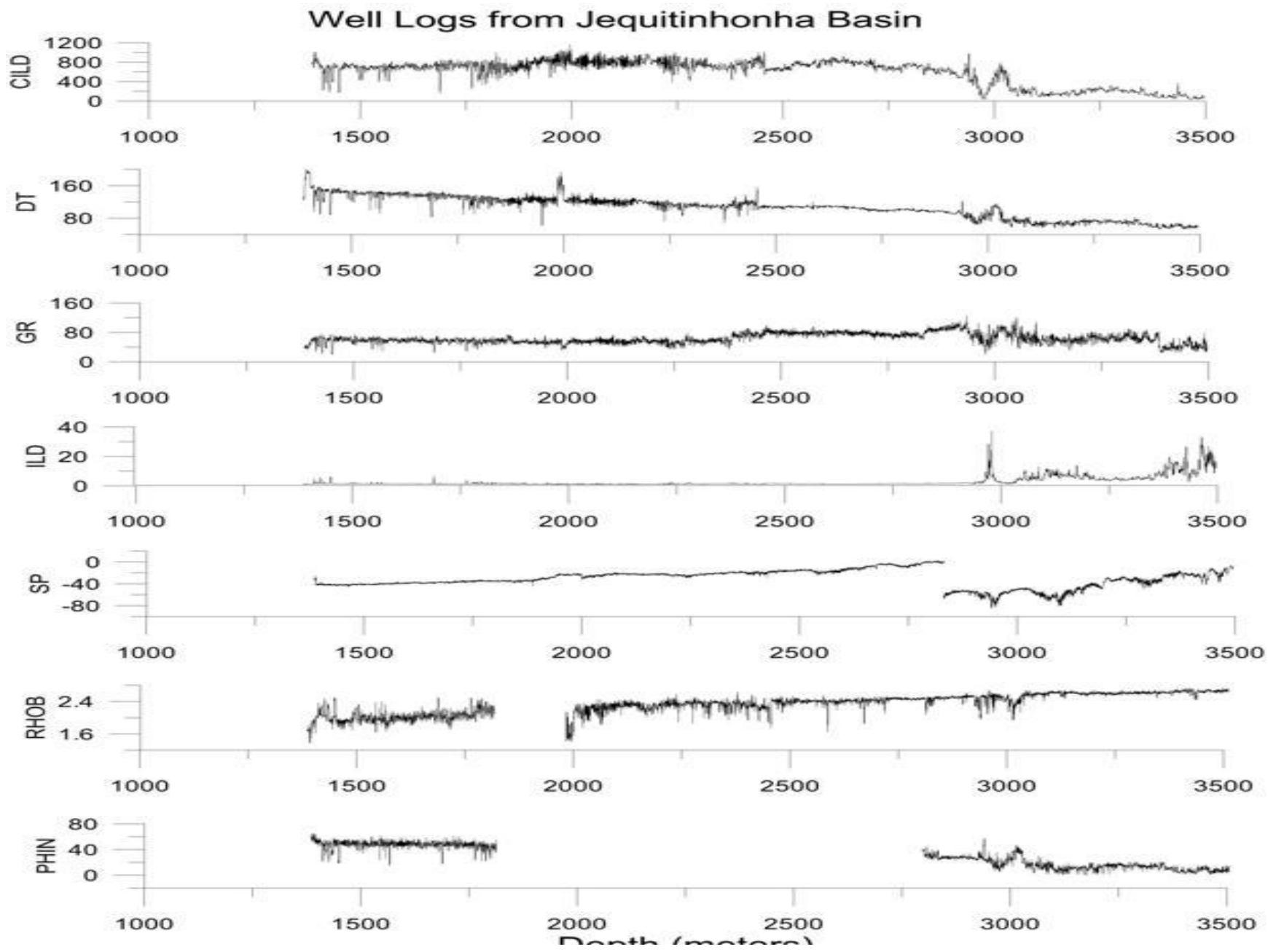

Figura 2 - Perfis de poço utilizados neste trabalho.

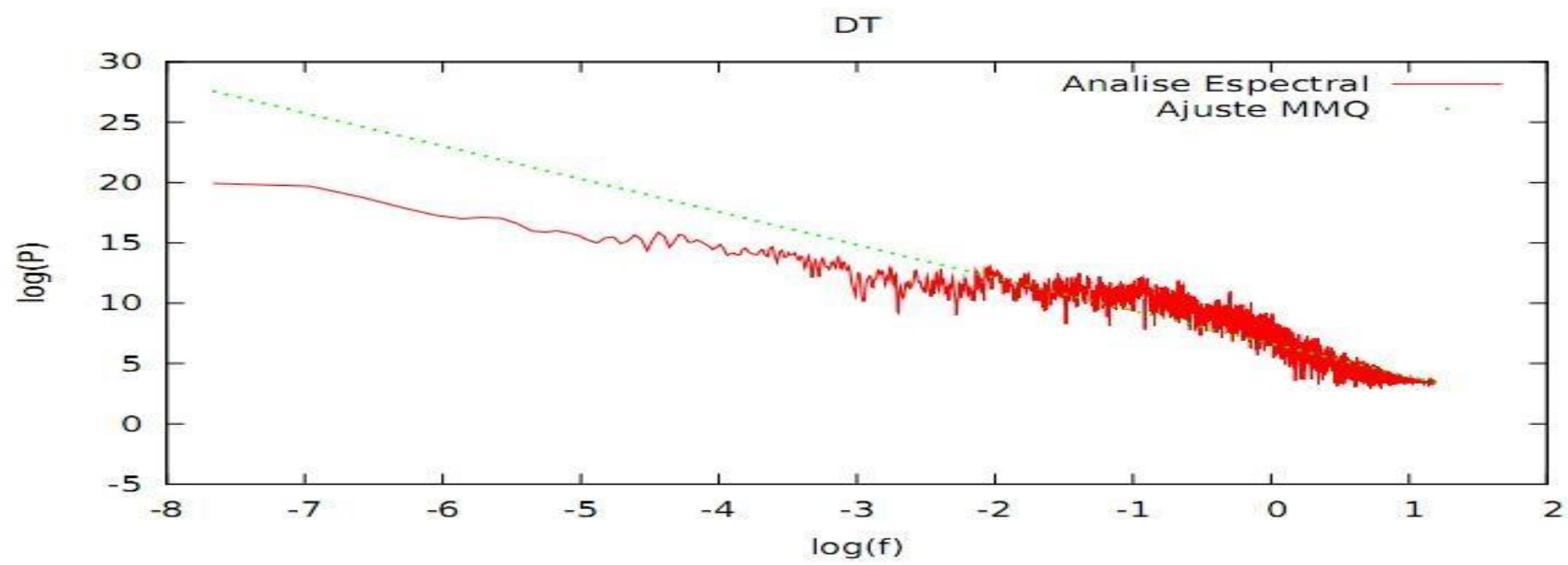

Figura 3 - Espectro de potência do tempo de trânsito da onda P (DT). Ajuste da curva pelo método dos mínimos quadrado IV Simpósio Brasileiro da SBGf - Brasília 2010 


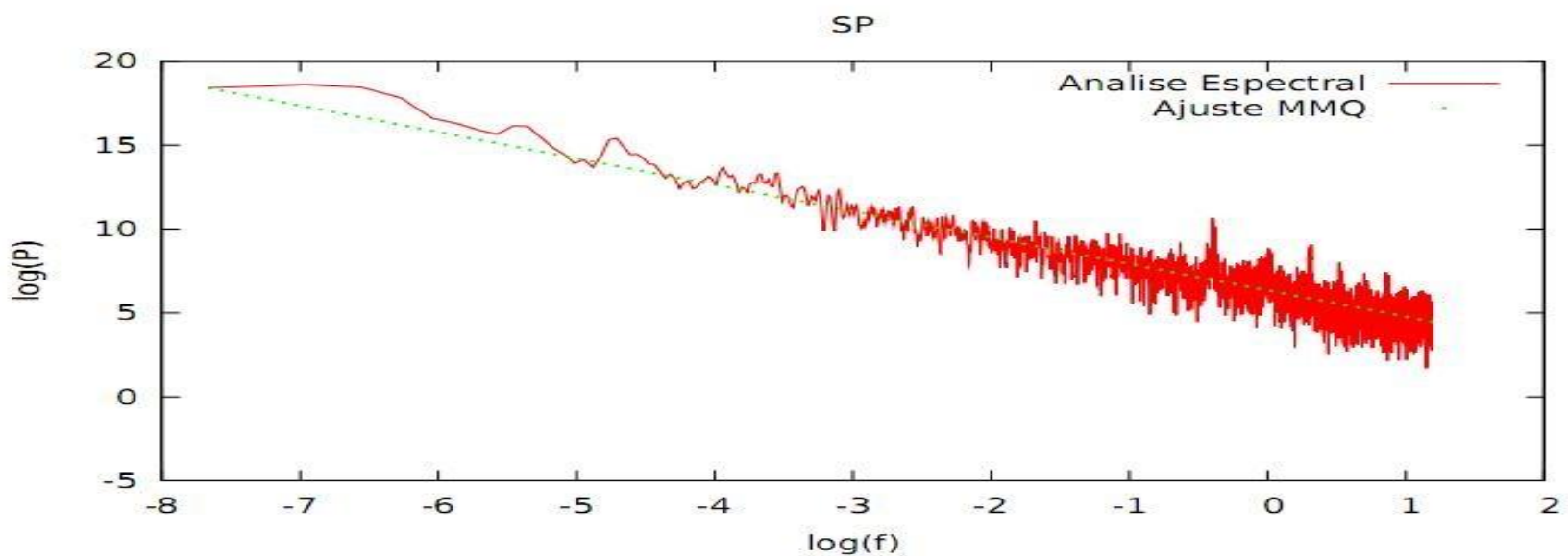

Figura 4 - Espectro de potência do potencial espontâneo (SP). Ajuste da curva pelo método dos mínimos quadrados.

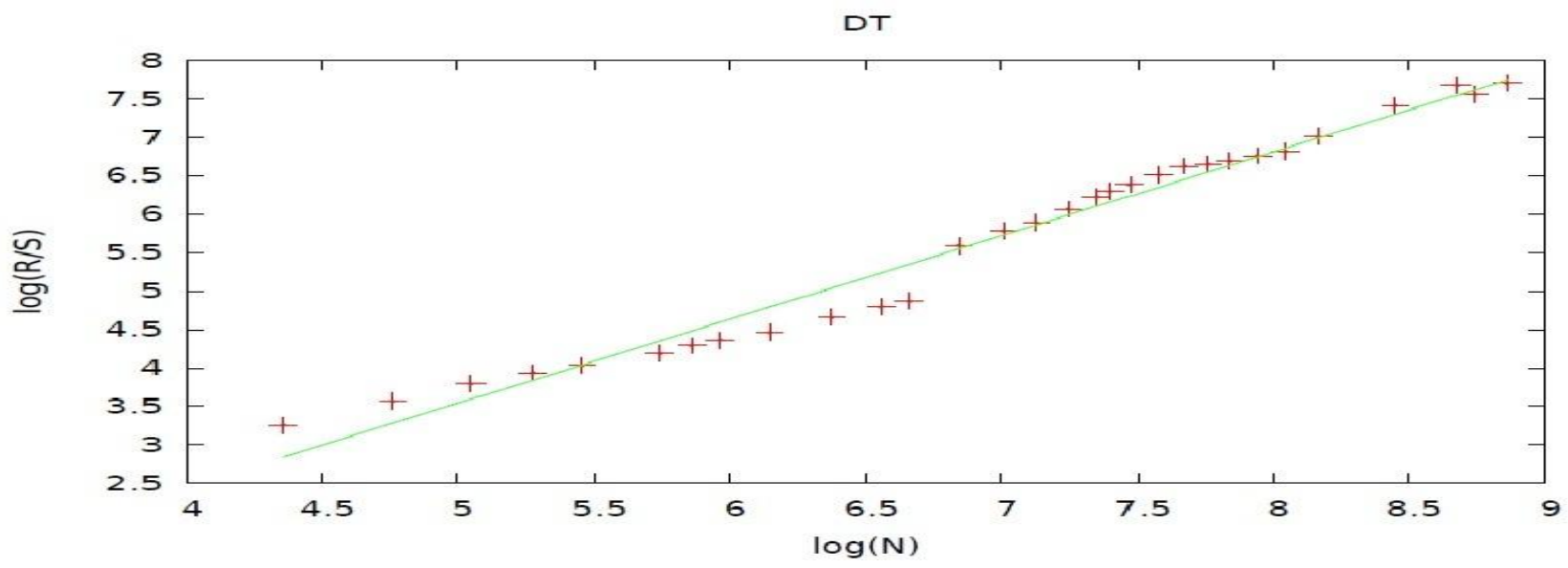

Figura 5 - Análise R/S do tempo de trânsito da onda $P(D T)$. Ajuste da curva pelo método dos mínimos quadrados.

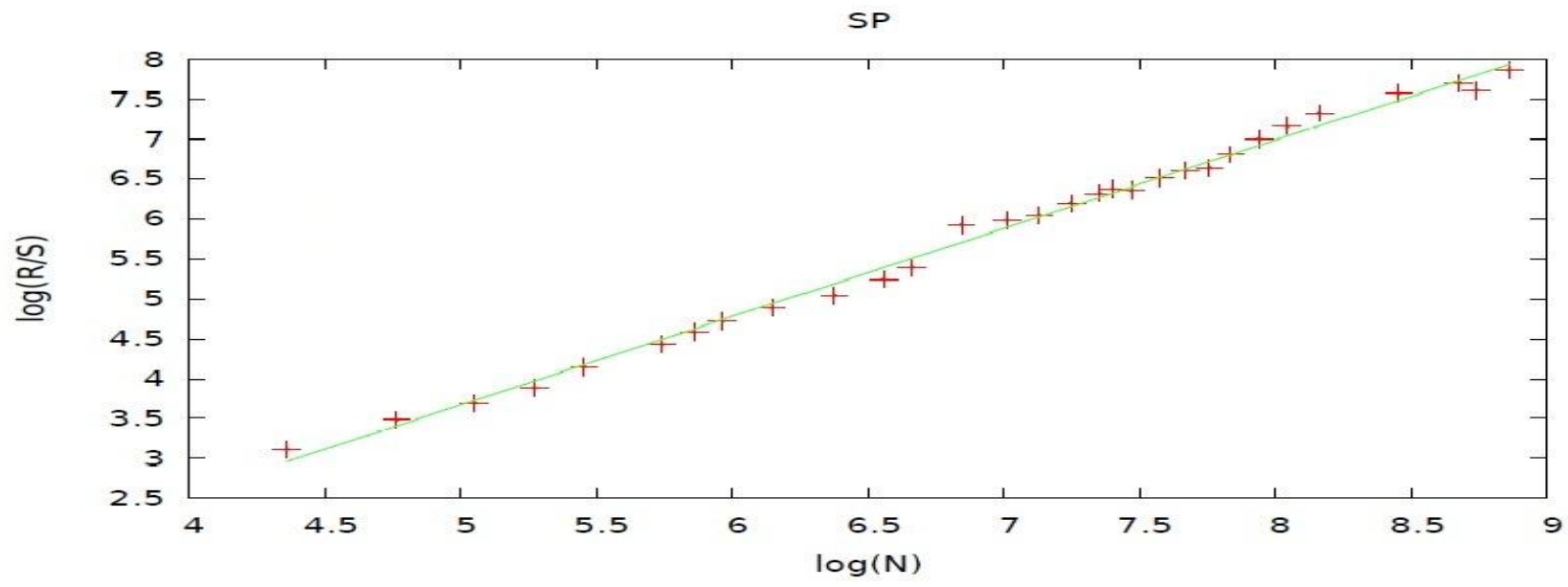

Figura 6 - Análise R/S do potencial espontâneo (SP). Ajuste da curva pelo método dos mínimos quadrados. 